\title{
Relationship between Echocardiographic Diastolic Indices and Invasively Measured Left Ventricular End-Diastolic Pressure in Chronic Atrial Fibrillation Patients with Preserved Left Ventricular Ejection Fraction
}

\author{
Jin Su Byun', Ji Hun Ahn" ${ }^{1, *}$, Ung Jeon ${ }^{2, *}$, Hye Yon Yu ${ }^{3}$ \\ 'Department of Internal Medicine, Soonchunhyang University Gumi Hospital, Gumi; ${ }^{2}$ Department of Internal Medicine, Soonchunhyang University Cheonan \\ Hospital; ${ }^{3}$ Department of Nursing, Soonchunhyang University College of Medicine, Cheonan, Korea
}

\begin{abstract}
Objective: Left ventricular (LV) filling pressure can be estimated using echocardiographic measurements, including the ratio of transmitral early peak flow velocity to tissue Doppler mitral annular motion velocity (E/e') during sinus rhythm. However, non-invasive echocardiographic estimation is complicated in patients with atrial fibrillation (AF). AF is associated with reduced survival in patients with heart failure with preserved LV ejection fraction (LVEF). The aim of this study was to investigate echocardiographic parameters for predicting LV filling pressure and diastolic function in chronic AF patients with preserved LVEF.

Methods: Clinical data, echocardiographic findings, and laboratory data were assessed retrospectively in 90 chronic AF patients with preserved LVEF who underwent diagnostic left-heart catheterization between January 2011 and September 2015. LV end-diastolic pressure (LVEDP) assessment and standard echocardiographic measurements were performed.

Results: The E/e' ratio was significantly correlated with LVEDP $(r=0.449, \mathrm{P}<0.001)$. Receiver operating characteristic analysis defined the optimal cut-off for E/e' as 13 (sensitivity, 88\%; specificity, 67\%) to predict elevated LVEDP ( $>15 \mathrm{~mm} \mathrm{Hg})$. E $(r=0.463, \mathrm{P}<0.001$ ) and E/propagation velocity $(\mathrm{Vp} ; \mathrm{r}=0.471, \mathrm{P}<0.001)$ were also correlated with LVEDP.E $>90 \mathrm{~cm} / \mathrm{sec}$ predicted elevated LVEDP with a sensitivity of $84 \%$ and a specificity of $70 \%$. Also, an E/Np $>1.6$ predicted elevated LVEDP with a sensitivity of $80 \%$ and a specificity of $72 \%$. Conclusion: $\mathrm{E}>90 \mathrm{~cm} / \mathrm{sec}, \mathrm{E} / \mathrm{e}^{\prime}>13$ and $\mathrm{E} / \mathrm{Vp}>1.6$ were suggestive of elevated LVEDP in these patients. Therefore, $\mathrm{E}, \mathrm{E} / \mathrm{e}^{\prime}$, and E/Np provide significant predictive value for LVEDP in chronic AF with preserved LVEF.
\end{abstract}

Keywords: Left ventricular filling pressure; Echocardiography; Atrial fibrillation

\section{INTRODUCTION}

Atrial fibrillation (AF) is the most common chronic arrhythmia associated with heart failure (HF) [1-4]. Approximately half of patients with HF show preserved left ventricular ejection fraction (LVEF), and AF is more prevalent in patients with HF with preserved rather than reduced LVEF [5-9].

AF with left ventricular (LV) diastolic dysfunction plays an important role in the prevalence and severity of HF, rather than AF with systolic dysfunction. AF is also associated with increased mortality in patients with HF with preserved LVEF as well as reduced LVEF [10-12]. Therefore, evaluation of LV diastolic function is important in chronic AF patients with preserved LVEF. However, conventional echocardiographic methods used to assess diastolic dysfunction in sinus rhythm cannot be readily applied in AF because of a lack of atrial contraction due to asynchronicity and variable heart rate $[4,13,14]$. Several echocardiographic and invasive studies examining diastolic dysfunction in AF have used the ratio of trans-mitral early peak flow velocity (E) to tissue Doppler mitral annular motion velocity (e'), the ratio of $\mathrm{E}$ to color M-mode
Correspondence to: Ji Hun Ahn

Department of Internal Medicine, Soonchunhyang University Gumi Hospital, 179 1(il)gongdan-ro, Gumi 39371, Korea

Tel: +82-54-468-9062, Fax: +82-54-468-3638, E-mail: jichoona@gmail.com

Co-correspondence to: Ung Jeon

Department of Internal Medicine, Soonchunhyang University Cheonan Hospital, 31 Suncheonhyang 6-gil, Dongnam-gu,

Cheonan 31151, Korea

Tel: +82-41-570-3893, Fax: +82-41-570-2843, E-mail: c120504@schmc.ac.kr

*These two corresponding authors contributed equally to this work.

Received: Nov. 9, 2018 / Accepted after revision: Dec. 10, 2018
(C) 2018 Soonchunhyang Medical Research Institute This is an Open Access article distributed under the terms of the Creative Commons Attribution Non-Commercial License (http://creativecommons.org/licenses/by-nc/4.0/) 
Echocardiographic Diastolic Indices Predict LV Filling Pressure in Chronic AF Patients with Preserved LVEF • Byun JS, et al.

LV propagation velocity (Vp), deceleration time (DT), and pulmonary capillary wedge pressure (PCWP) by measurement devices [13-18]. However, the association between non-invasive echocardiographic estimation of LV filling pressure and invasive measurement of LV filling pressure remains unclear and is a challenging issue in chronic AF patients with preserved LVEF.

The purpose of this study was to investigate the correlation between non-invasive echocardiographic parameters and the invasive measurement of LV filling pressure and to identify which echocardiographic parameters can predict LV diastolic function in chronic AF patients with preserved LVEF.

\section{MATERIALS AND METHODS}

\section{Patient population}

The medical records of 90 consecutive patients who presented with chronic AF and preserved LVEF from January 2011 to September 2015 were evaluated retrospectively. All patients had their conditions confirmed on more than two consecutive electrocardiograms over a 3-month period. Preserved LVEF was defined as greater than 50\% LVEF with no regional wall motion abnormalities evident on echocardiography. Patients were excluded from the study based on the following criteria: paroxysmal AF, rheumatic disease, prosthetic mitral valves, mitral stenosis, aortic valve stenosis, severe valvular insufficiency, pulmonary hypertension, or a history of pulmonary embolism.

The institutional review board of Soonchunhyang University Gumi Hospital approved the study protocol (IRB approval no., 2015-14). Written informed consent was obtained from all participating patients.

\section{Echocardiography}

Standard transthoracic two-dimensional, M-mode, and Doppler echocardiograms were performed in the left lateral decubitus position with GE Vivid 7 instrument (GE Healthcare, Milwaukee, WI, USA) equipped with M4S transducers within 24 hours before cardiac catheterization. Measurements were taken according to the recommendations of the American Society of Echocardiography [19]. We measured LV end-diastolic and end-systolic volumes and LVEF using the modified biplane Simpson's method, and left atrial (LA) volume by the prolate ellipse method. E, e', and Vp were measured using the dual Doppler imaging method. We calculated right atrial (RA) pressure from the inferior vena cava diameter and the presence of inspiratory collapse. Pulmonary artery systolic pressure (PASP) was determined from combining the tricuspid regurgitation gradient and calculated RA pressure in the absence of a gradient of pulmonary valve flow velocity [20]. The measurements reported are an average of five consecutive cardiac cycles. All echocardiographic measurements and estimations were performed without knowledge of invasive measurement of LV filling pressure.

\section{Brain natriuretic peptide measurements}

Blood samples were obtained for measurement of brain natriuretic peptide (BNP) within 24 hours of echocardiographic examination. BNP was measured by chemiluminescence enzyme immunoassay.

\section{Hemodynamic measurements}

Left heart catheterization was performed via radial or femoral approach. The measurement of LV end-diastolic pressure (LVEDP) was performed with a pig-tail catheter before coronary angiography. Measurements from 10 consecutive cardiac beats

Table 1. Baseline clinical characteristics of the patients $(n=90)$

\begin{tabular}{lc}
\hline Patient characteristic & Value \\
\hline Age (yr) & $68.8 \pm 10.1$ \\
Sex & \\
Male & 47 \\
Female & 43 \\
New York Heart Association class & \\
1 & $37(41.1)$ \\
2 & $26(28.8)$ \\
3 & $15(16.6)$ \\
4 & $3(3.3)$ \\
Clinical history & \\
Coronary artery disease & $26(28.8)$ \\
Myocardial infarction & $7(7.7)$ \\
Hypertension & $47(52.2)$ \\
Diabetes mellitus & $22(24.4)$ \\
Hyperlipidemia & $31(34.4)$ \\
Lone atrial fibrillation & $26(28.8)$ \\
Medication & \\
Angiotensin-converting enzyme inhibitor & $3(5.0)$ \\
Angiotensin receptor blocker & $31(34.4)$ \\
Beta-blocker & $44(48.8)$ \\
Calcium channel blocker & $33(36.6)$ \\
Digoxin & $35(38.8)$ \\
Diuretics & $38(42.2)$ \\
\hline
\end{tabular}

Values are presented as mean \pm standard deviation or number $(\%)$. 
Byun JS, et al. • Echocardiographic Diastolic Indices Predict LV Filling Pressure in Chronic AF Patients with Preserved LVEF

were recorded by an investigator unaware of the echocardiographic data and averaged to obtain a final measurement.

\section{Statistical analysis}

Data are presented as the mean \pm standard deviation. Comparisons between variables were performed using the t-test and Fischer's exact test. Correlations between echocardiographic parameters and LVEDP were determined using linear regression analysis. The diagnostic accuracies of echocardiographic parameters were compared by receiver operating characteristic (ROC) curve analysis. Statistical significance was defined as $\mathrm{P}<0.05$. All statistical analyses were performed using the IBM SPSS ver. 20.0 software package (IBM Corp., Armonk, NY, USA).

\section{Reproducibility}

All echocardiographic measurements were performed randomly by one observer and repeated on 2 separate days by two observers who were blinded to each other's measurement results and study time points, which allowed us to assess intra- and inter-observer reproducibility.

\section{RESULTS}

\section{Baseline and echocardiographic characteristics}

The patients were divided into two groups: group 1 included 52 patients with normal LVEDP and group 2 included 38 patients with increased LVEDP (>15 mm Hg). The baseline characteristics of the patients are presented in Table 1 . The mean age of the patients was $68.8 \pm 10.1$ years, and 47 patients were male. Eighteen patients had New York Heart Association class 3 or 4 of HF, 33 had coronary artery disease or prior myocardial infarction, and 26 patients had only AF. There were no significant differences between the two groups in age, sex, medical history, or drug history. The echocardiographic parameters, hemodynamic measurements, and laboratory data are presented in Table 2. The mean LVEF of all patients was $60.9 \pm 5.2$, and the mean heart rate was $82.2 \pm$

Table 2. Echocardiographic, hemodynamic, and laboratory data

\begin{tabular}{|c|c|c|c|c|}
\hline Variable & All patients $(n=90)$ & Group $1(n=52)$ & Group 2 ( $n=38$ ) & P-value \\
\hline \multicolumn{5}{|l|}{ Echocardiographic index } \\
\hline LV end-diastolic dimension (mm) & $51.2 \pm 4.9$ & $50.3 \pm 4.6$ & $52.3 \pm 5.2$ & 0.06 \\
\hline LV end-systolic dimension (mm) & $34.4 \pm 4.3$ & $33.6 \pm 3.6$ & $35.4 \pm 4.9$ & 0.05 \\
\hline Interventricular septum (mm) & $8.6 \pm 2.0$ & $8.6 \pm 1.8$ & $8.7 \pm 2.2$ & 0.87 \\
\hline LV posterior free wall (mm) & $8.5 \pm 1.5$ & $8.4 \pm 1.1$ & $8.7 \pm 1.8$ & 0.29 \\
\hline $\mathrm{LV}$ mass index $\left(\mathrm{gm} / \mathrm{m}^{2}\right)$ & $96.1 \pm 28.7$ & $92.0 \pm 25.2$ & $101.7 \pm 32.3$ & 0.11 \\
\hline Relative wall thickness & $0.3 \pm 0.1$ & $0.3 \pm 0.1$ & $0.3 \pm 0.1$ & 0.83 \\
\hline Ejection fraction $(\%)$ & $60.9 \pm 5.2$ & $61.4 \pm 4.5$ & $60.2 \pm 6.1$ & 0.28 \\
\hline Left atrial volume index $\left(\mathrm{mL} / \mathrm{m}^{2}\right)$ & $43.2 \pm 16.8$ & $38.4 \pm 11.9$ & $49.8 \pm 20.2$ & $<0.01$ \\
\hline Mitral E (m/sec) & $94.8 \pm 24.5$ & $86.2 \pm 18.5$ & $106.5 \pm 27.1$ & $<0.01$ \\
\hline Deceleration time (msec) & $168.2 \pm 33.8$ & $170.2 \pm 32.2$ & $165.3 \pm 36.2$ & 0.50 \\
\hline Pulmonary arterial systolic pressure $(\mathrm{mm} \mathrm{Hg})$ & $32.9 \pm 11.0$ & $31.4 \pm 9.2$ & $37.2 \pm 12.5$ & $<0.05$ \\
\hline Septal e' (m/sec) & $7.0 \pm 1.9$ & $7.3 \pm 2.2$ & $6.7 \pm 1.5$ & 0.16 \\
\hline$E / e^{\prime}$ & $14.2 \pm 5.3$ & $12.5 \pm 4.5$ & $16.6 \pm 5.6$ & $<0.01$ \\
\hline$V p(m / s e c)$ & $57.9 \pm 15.7$ & $61.7 \pm 15.6$ & $52.5 \pm 14.6$ & $<0.05$ \\
\hline $\mathrm{E} / \mathrm{Np}$ & $1.8 \pm 0.8$ & $1.5 \pm 0.5$ & $2.3 \pm 0.9$ & $<0.01$ \\
\hline \multicolumn{5}{|l|}{ Catheterization and laboratory data } \\
\hline $\operatorname{LVEDP}(\mathrm{mm} \mathrm{Hg})$ & $14.9 \pm 4.5$ & $11.9 \pm 2.3$ & $19.2 \pm 3.1$ & $<0.01$ \\
\hline Minimal LV pressure & $10.6 \pm 4.7$ & $8.2 \pm 2.7$ & $13.9 \pm 4.9$ & $<0.01$ \\
\hline Systolic blood pressure (mm Hg) & $123.3 \pm 18.3$ & $125.0 \pm 18.2$ & $120.9 \pm 18.3$ & 0.29 \\
\hline Diastolic blood pressure $(\mathrm{mm} \mathrm{Hg})$ & $77.1 \pm 12.2$ & $78.7 \pm 11.6$ & $75.0 \pm 12.9$ & 0.15 \\
\hline Heart rate (beats/min) & $82.2 \pm 22.4$ & $81.8 \pm 17.7$ & $92.9 \pm 27.9$ & 0.82 \\
\hline Brain natriuretic peptide $(\mathrm{pg} / \mathrm{mL})$ & $352.1 \pm 528.9$ & $341.8 \pm 646.0$ & $366.2 \pm 312.0$ & 0.83 \\
\hline
\end{tabular}

Values are presented as mean \pm standard deviation. Group 1: LVEDP < $15 \mathrm{~mm} \mathrm{Hg}$; group 2: LVEDP > $15 \mathrm{~mm} \mathrm{Hg}$.

$\mathrm{LV}$, left ventricular; Vp, propagation velocity; LVEDP, left ventricular end diastolic pressure; 
22.4 beats/min. LVEF was preserved, and there were no significant differences between the two groups. Heart rate was well controlled in both groups. The mean LVEDP was $14.9 \pm 4.5 \mathrm{~mm} \mathrm{Hg}$ and the mean minimal LV pressure was $10.6 \pm 4.7 \mathrm{~mm} \mathrm{Hg}$. The LA volume index, mitral E, PASP, E/e', and E/Vp were significantly higher and the Vp was significantly lower in group 2 than in group 1 (LVEDP > $15 \mathrm{~mm} \mathrm{Hg}$ ). However, there were no significant differences between the two groups in terms of EF, DT, HR, or BNP.

\section{Relationship between left ventricular filling pressure and echocardiographic parameters}

$\mathrm{E}(\mathrm{r}=0.463, \mathrm{P}<0.001), \mathrm{E} / \mathrm{e}^{\prime}(\mathrm{r}=0.449, \mathrm{P}<0.001)$, and $\mathrm{E} / \mathrm{Vp}$ $(\mathrm{r}=0.471, \mathrm{P}<0.001)$ demonstrated significant linear correlations with LVEDP. The PASP $(r=0.422, \mathrm{P}<0.001)$ and LA volume in$\operatorname{dex}(r=0.313, P=0.003)$ values were positively correlated with LVEDP (Fig. 1). Also, E ( $\mathrm{r}=0.267, \mathrm{P}=0.011), \mathrm{E} / \mathrm{e}^{\prime}(\mathrm{r}=0.262, \mathrm{P}=$ $0.013), \mathrm{E} / \mathrm{Vp}(\mathrm{r}=0.325, \mathrm{P}=0.011)$, PASP $(\mathrm{r}=0.289, \mathrm{P}=0.006)$, and
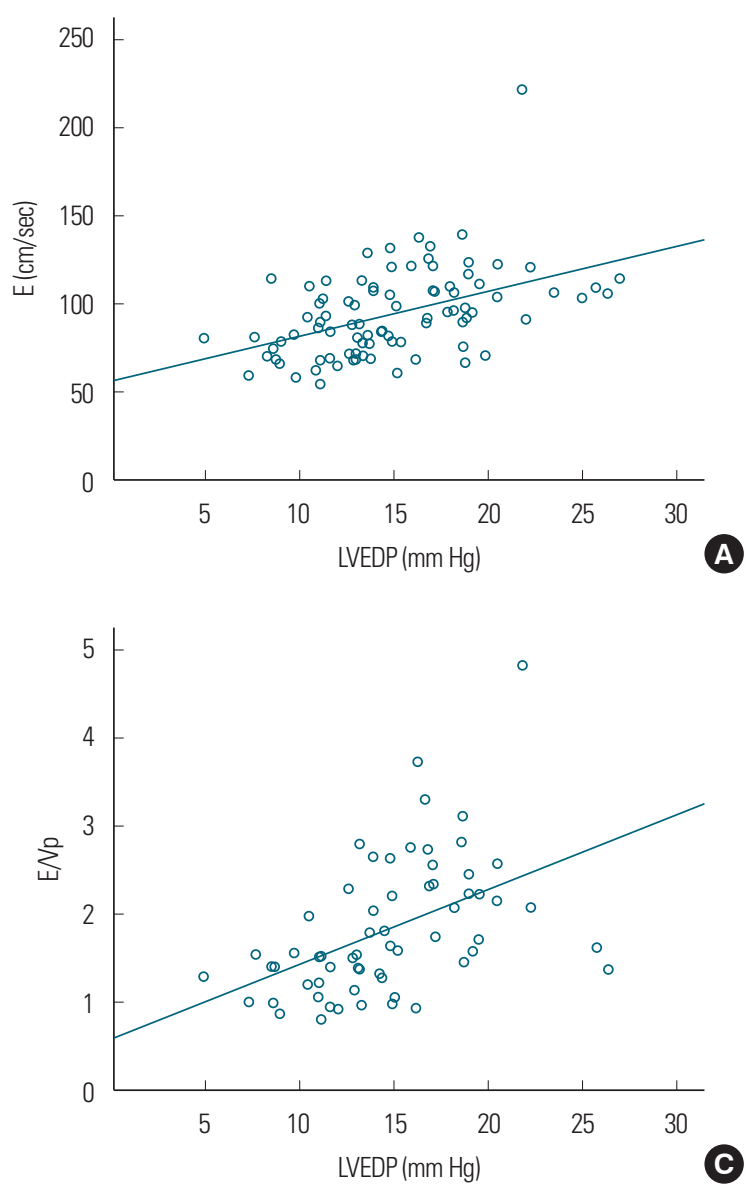

LA volume index $(r=0.247, \mathrm{P}=0.019)$ demonstrated positive linear correlations with minimal LV pressure (Fig. 2). The predictive values of E, E/e', and E/Vp for the detection of LVEDP > $15 \mathrm{~mm}$ Hg were determined with ROC curve analysis (Fig. 3) using the area under the curve (E, 0.782; E/e', 0.790; E/Vp, 0.799). The optimal cutoff value of E/e' was 13 (sensitivity, $88 \%$; specificity, 67\%) to predict $>15 \mathrm{~mm} \mathrm{Hg}$ LVEDP. E $>90 \mathrm{~cm} / \mathrm{sec}$ predicted elevated LVEDP ( $>15 \mathrm{~mm} \mathrm{Hg}$ ) with a sensitivity of $84 \%$ and a specificity of $70 \%$. Also, E/Vp > 1.6 predicted elevated LVEDP (>15 mm Hg) with a sensitivity of $80 \%$ and a specificity of $72 \%$ (Fig. 3).

\section{DISCUSSION}

Diastolic dysfunction plays an important role in the development of e related with AF $[3,4]$. Patients HF with preserved LVEF comprise approximately $40 \%-50 \%$ of the total HF population, and $\mathrm{AF}$ is more likely to occur in these patients [5-9]. AF was associat-
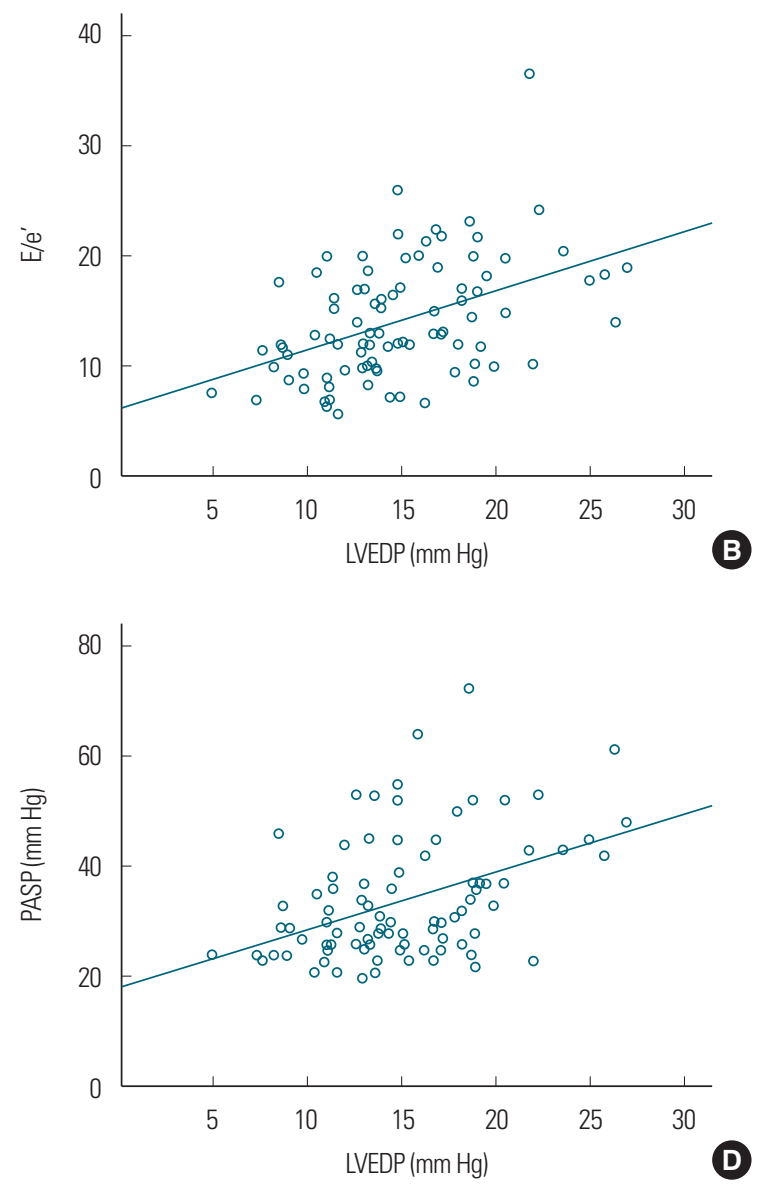

Fig. 1. Relationship between echocardiographic parameters and LVEDP. (A) $E: r=0.464, P<0.001$; (B) $E / e^{\prime}: r=0.449, P<0.001 ;(C) E / N p: r=0.471, P<0.05$; and (D) PASP: $r=0.503, P<0.001$. LVEDP, left ventricular end diastolic pressure; $V p$, propagation velocity; PASP, pulmonary arterial systolic pressure. 

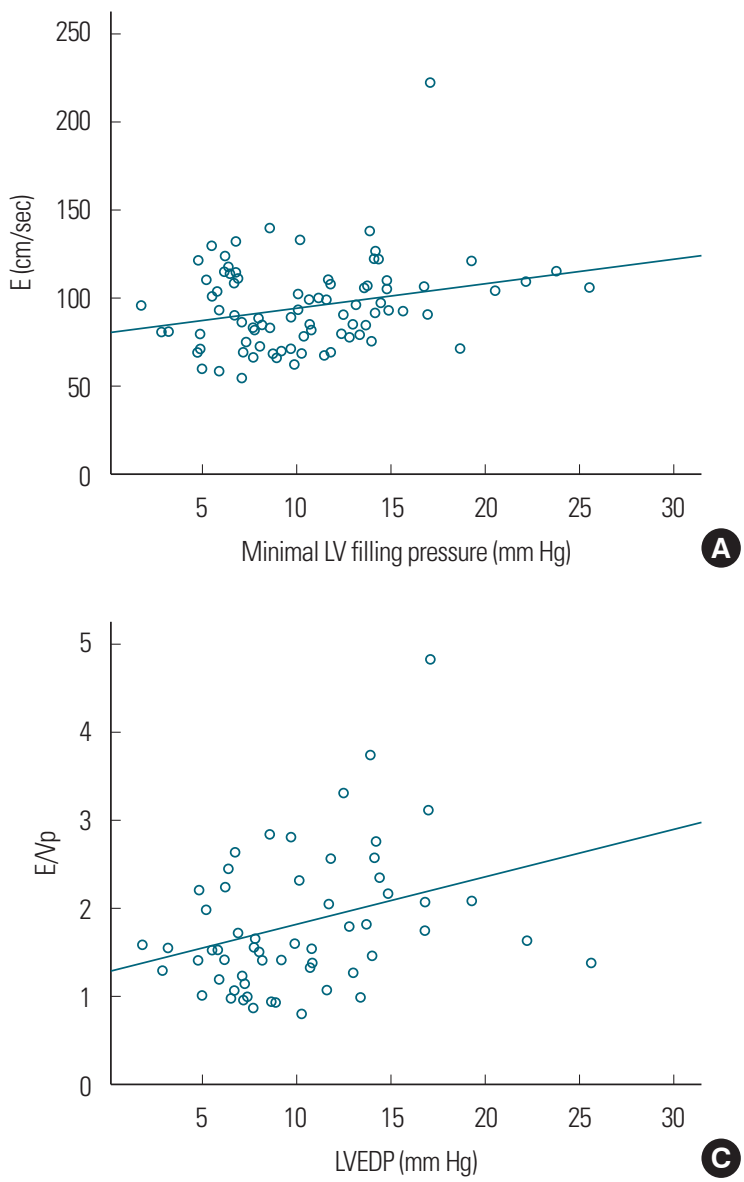
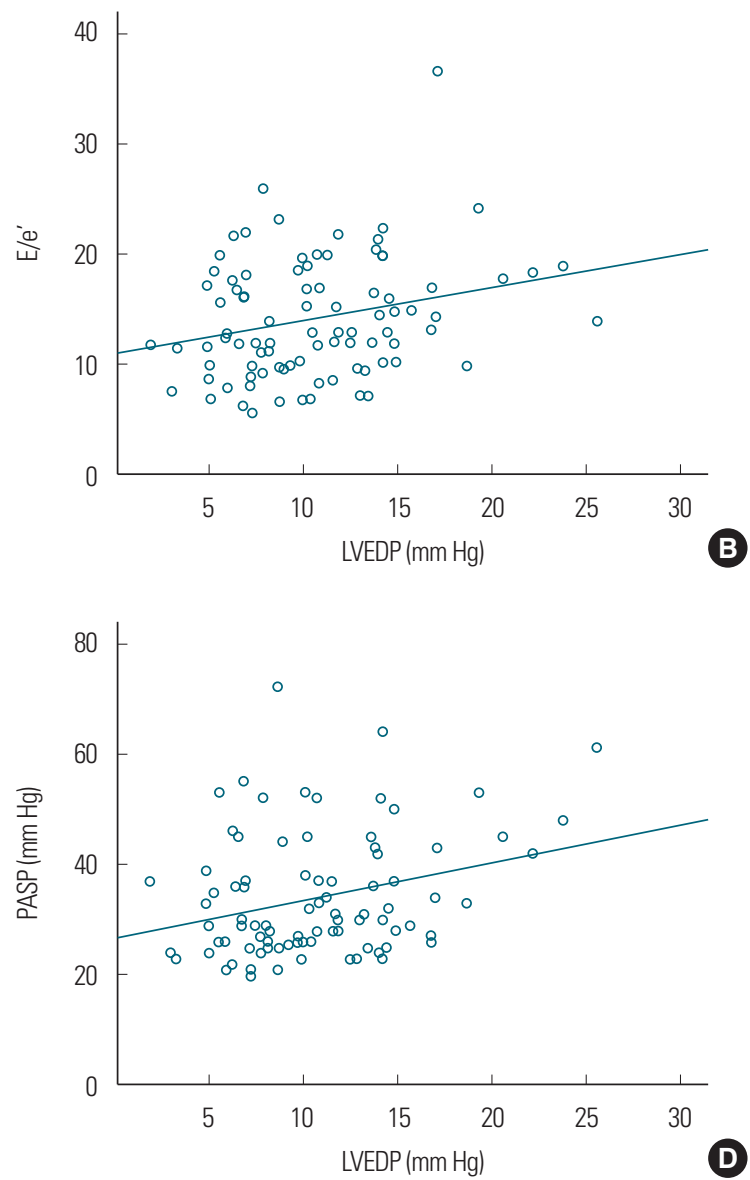

Fig. 2. Relationship between echocardiographic parameters and minimal LV filling pressure. (A) $E: r=0.267, P=0.011 ;(B) E / e^{\prime}: r=0.262, P=0.013 ;(C) E / N p: r=0.325$, $P=0.011$; and (D) PASP: $r=0.289, P=0.006$. LV, left ventricular; LVEDP, left ventricular end diastolic pressure; Vp, propagation velocity; PASP, pulmonary arterial systolic pressure.

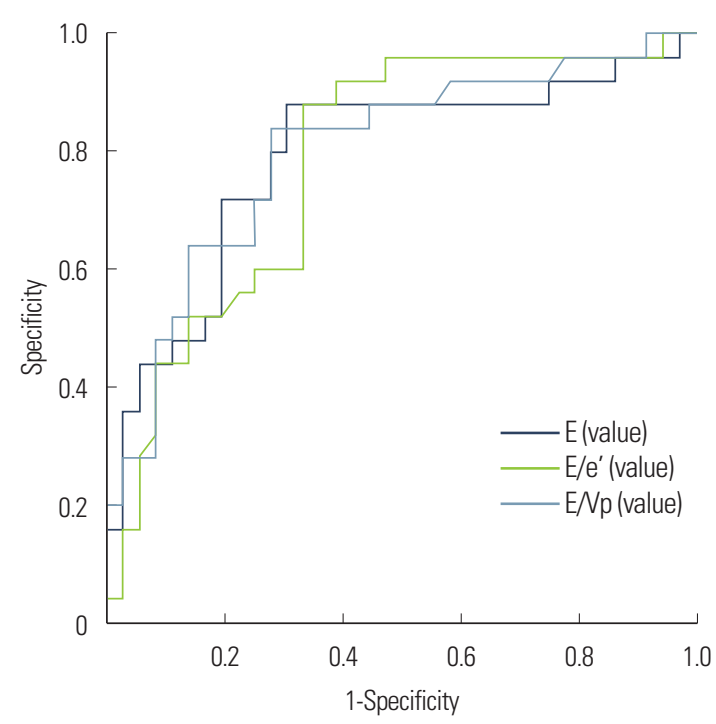

Fig. 3. Receiver operating characteristic curve for predicting elevated LVEDP. The area under the curve for using E, E/e', and E/Np for differentiating LVEDP $>15 \mathrm{~mm} \mathrm{Hg}$ from that of $\leq 15 \mathrm{~mm} \mathrm{Hg}$ was $0.782,0.790$, and 0.799 , respectively. LVEDP, left ventricular end diastolic pressure; Vp, propagation velocity. ed with reduced survival in patients with HF with preserved LVEF [10-12]. Therefore, evaluation of LV diastolic function is important in chronic AF patients with preserved LVEF to prevent worsening of HF. Until recently, several studies have evaluated the relationship between diastolic dysfunction and echocardiographic parameters in the setting of AF [13-18]. However, conventional echocardiographic methods used to assess diastolic dysfunction in sinus rhythm cannot be readily applied in AF because of the lack of atrial contraction due to atrial contractile asynchronicity and variability in heart rate $[4,13,14]$. The aim of this study was to investigate echocardiographic parameters for predicting LV filling pressure and diastolic function in chronic AF patients with preserved LVEF.

In the present study, the main findings were: (1) LVEDP was elevated in chronic, persistent AF patients with preserved LV systolic function, and (2) E, E/e', and E/Vp had significant predictive value for LVEDP in chronic AF patients with preserved LVEF. 
Echocardiographic Diastolic Indices Predict LV Filling Pressure in Chronic AF Patients with Preserved LVEF • Byun JS, et al.

\section{Elevated left ventricular filling pressure in chronic atrial fibrillation patients}

In $\mathrm{AF}$, atrioventricular asynchrony and irregular ventricular response can adversely affect ventricular function, leading to a decrease in cardiac output and impairment of diastolic filling $[1,4,10,13]$. Moreover, in chronic AF, these characteristics can result in fluid retention and LVEDP elevation. In the current study, we demonstrated the elevation of LVEDP by invasive measurement of LV filling pressure (14.9 $\pm 4.5 \mathrm{~mm} \mathrm{Hg}$ ), which showed the impact of AF on diastolic dysfunction in chronic AF patients with preserved LVEF.

\section{The relationship between echocardiographic parameters and left ventricular filling pressure}

The E velocity is the early LV filling velocity and is influenced by LV relaxation and LA pressure $[13,15]$. Several studies have demonstrated weak to moderate correlations between peak $\mathrm{E}$ and LV filling pressure via measurement of PCWP in patients with AF, but its predictive value was not obvious $[13,16,17]$. In our study, modest correlations were found between E and LVEDP, as well as minimal LV pressure, by direct measurement of LV filling pressure; here, $\mathrm{E}>90 \mathrm{~cm} / \mathrm{sec}$ was the predictive value for detection of elevated LVEDP (>15 mm Hg). Peak E velocity is associated either with normal LV relaxation and low LV filling pressure or abnormal LV relaxation and elevated LA pressure $[13,15]$. Our results seemed to reflect the latter condition.

Prior studies on the E/Vp ratio in patients with AF demonstrated a modest correlation with LV filling pressure, and presented a predictive value associated with increased LVEDP [13,21]. A previous study demonstrated that E/VP $>1.4$ predicted an elevated PCWP ( $\geq 15 \mathrm{~mm} \mathrm{Hg}$ ) with a sensitivity of $72 \%$ and a specificity of $100 \%$ [13]; another study found that mean $\mathrm{E} / \mathrm{Vp} \geq 1.7$ predicted increased BNP ( $\geq 200 \mathrm{pg} / \mathrm{mL}$ ) with a sensitivity of $80 \%$ and a specificity of $84 \%$ [21]. Our results were similar to those of the above-mentioned studies and demonstrated that $\mathrm{E} / \mathrm{Vp}$ is modestly correlated with LV filling pressure and has good predictive value ( $>1.6$ ) in the detection of elevated $\operatorname{LVEDP}(>15 \mathrm{~mm} \mathrm{Hg}$ ), but we did not find a relationship between E/Vp and BNP. A rapid decrease in $\mathrm{Vp}$, as well as an increase in peak E velocity, was associated with elevated LVEDP and impaired LV relaxation [22]. So, E/Vp increases with HF deterioration and is a more sensitive measurement than is E alone. However, in our study, the predictive value of $\mathrm{E}$ and $\mathrm{E} / \mathrm{Vp}$ was similar in patients with preserved LVEF. So, we posit that our study population consisted of patients with less advanced $H F$. These characteristics may influence the relationship between $\mathrm{E} / \mathrm{Vp}$ and BNP, and the predictive value of $\mathrm{E}$ and $\mathrm{E} / \mathrm{Vp}$.

Theoretically, E/e' is the preferred measure in patients with preserved LVEF because it is less variable than E/Vp [23]. In a previous study, E/e' was shown to be a reasonably sensitive and easy to measure parameter for the prediction of diastolic dysfunction in patients with AF; E/e' $\geq 11$ predicted elevated LV filling pressure ( $\geq 15 \mathrm{~mm} \mathrm{Hg}$ ) with a sensitivity of $75 \%$ and a specificity of $93 \%$ [14]. However, that study had a small number of patients with AF and variable characteristics that could affect the value of E/e' for predicting elevated LVEDP. We used a larger study population that consisted of chronic AF patients with preserved LVEF and direct measurements of LV filling pressure. Our study demonstrated a good correlation between E/e' and LVEDP, as well as minimal LV pressure, and shows the clinical usefulness of $E / e^{\prime}$ in predicting elevated LV filling pressure. Recently, some studies reported that E/e' recorded using dual Doppler echocardiography had a good correlation and predictive value for PCWP and BNP [24,25]. Other studies suggest that lateral mitral E/e' is more highly correlated with PCWP in patients with preserved LVEF $[23,26]$.

Our study showed that the left atrial volume index (LAVI) and PASP had weak correlations and no predictive value for LV filling pressure in chronic AF patients with preserved LVEF. LA enlargement occurs frequently in AF, and the LAVI is influenced by LV filling pressure. However, some previous studies also observed no relationship between LAVI and LV filling pressure and did not find clinical usefulness of this measurement for estimating LV filling pressure [27-29]. Several studies have demonstrated a relationship between increased PASP and LV diastolic dysfunction in patients with myocardial infarction or preserved LV systolic function with the sinus rhythm [30,31]. Furthermore, Neuman et al. [32] showed a good correlation between PASP and LVEDP in patients with chronic AF. However, in our study that consisted of chronic AF patients with 'preserved EF', PASP was observed to be only weakly correlated with LVEDP. We found that LVEDP had a weak impact on PASP, when LVEF was preserved, unlike what has been reported in previous studies.

Echocardiographic evaluations of diastolic dysfunction are difficult and often unclear in patients with AF because the characteristics of AF have not been extensively studied using direct measurement of LV filling pressure. In our study, we examined the elevation of LVEDP by direct measurement of LV filling pressure 
Byun JS, et al. • Echocardiographic Diastolic Indices Predict LV Filling Pressure in Chronic AF Patients with Preserved LVEF

and demonstrated the relationship and predictive value of simple volume-dependent echocardiographic parameters for LV filling pressure in chronic AF patients with preserved LVEF.

\section{Limitations}

Our study had a few limitations. First, we obtained data on LV filling pressure from a small number of patients only. The patients were elderly, had controlled heart rates, and their population was composed of a relatively homogeneous and unrepresentative cohort of chronic AF patients with preserved LVEF. Second, echocardiographic measurements and LV catheterization were performed at different times in the patients, which could impact the correlation between echocardiographic parameters and LV filling pressure. Therefore, further investigation into the prognostic value and serial changes in echocardiographic parameters is warranted.

\section{Conclusions}

In our study, we observed that E, E/Vp, and E/e' were well correlated with LV filling pressure in patients with chronic AF and preserved LVEF. Moreover, our findings demonstrated the usefulness of E, E/Vp, and E/e' for estimating LV filling pressure in these patients. $\mathrm{E}>90 \mathrm{~cm} / \mathrm{sec}, \mathrm{E} / \mathrm{Vp}>1.6$, and $\mathrm{E} / \mathrm{e}^{\prime}>13$ were suggestive of elevated LVEDP (>15 mm Hg) in patients with chronic AF with preserved LVEF.

\section{REFERENCES}

1. Maisel WH, Stevenson LW. Atrial fibrillation in heart failure: epidemiology, pathophysiology, and rationale for therapy. Am J Cardiol 2003;91: 2D-8D.

2. Gersh BJ, Tsang TS, Seward JB. The changing epidemiology and natural history of nonvalvular atrial fibrillation: clinical implications. Trans Am Clin Climatol Assoc 2004;115:149-59.

3. Deedwania PC, Lardizabal JA. Atrial fibrillation in heart failure: a comprehensive review. Am J Med 2010;123:198-204.

4. Caldwell JC, Mamas MA. Heart failure, diastolic dysfunction and atrial fibrillation; mechanistic insight of a complex inter-relationship. Heart Fail Rev 2012;17:27-33.

5. Aljaroudi W, Alraies MC, Halley C, Rodriguez L, Grimm RA, Thomas JD, et al. Impact of progression of diastolic dysfunction on mortality in patients with normal ejection fraction. Circulation 2012;125:782-8.

6. Mozaffarian D, Benjamin EJ, Go AS, Arnett DK, Blaha MJ, Cushman M, et al. Heart disease and stroke statistics: 2015 update: a report from the American Heart Association. Circulation 2015;131:e29-322.

7. Owan TE, Hodge DO, Herges RM, Jacobsen SJ, Roger VL, Redfield MM. Trends in prevalence and outcome of heart failure with preserved ejection fraction. N Engl J Med 2006;355:251-9.

8. Hogg K, Swedberg K, McMurray J. Heart failure with preserved left ventricular systolic function; epidemiology, clinical characteristics, and prog- nosis. J Am Coll Cardiol 2004;43:317-27.

9. Bhatia RS, Tu JV, Lee DS, Austin PC, Fang J, Haouzi A, et al. Outcome of heart failure with preserved ejection fraction in a population-based study. N Engl J Med 2006;355:260-9.

10. Clark DM, Plumb VJ, Epstein AE, Kay GN. Hemodynamic effects of an irregular sequence of ventricular cycle lengths during atrial fibrillation. J Am Coll Cardiol 1997;30:1039-45.

11. Zile MR, Baicu CF, Gaasch WH. Diastolic heart failure: abnormalities in active relaxation and passive stiffness of the left ventricle. N Engl J Med 2004;350:1953-9.

12. Zile MR, Lewinter MM. Left ventricular end-diastolic volume is normal in patients with heart failure and a normal ejection fraction: a renewed consensus in diastolic heart failure. J Am Coll Cardiol 2007;49:982-5.

13. Nagueh SF, Kopelen HA, Quinones MA. Assessment of left ventricular filling pressures by Doppler in the presence of atrial fibrillation. Circulation 1996;94:2138-45.

14. Sohn DW, Song JM, Zo JH, Chai IH, Kim HS, Chun HG, et al. Mitral annulus velocity in the evaluation of left ventricular diastolic function in atrial fibrillation. J Am Soc Echocardiogr 1999;12:927-31.

15. Al-Omari MA, Finstuen J, Appleton CP, Barnes ME, Tsang TS. Echocardiographic assessment of left ventricular diastolic function and filling pressure in atrial fibrillation. Am J Cardiol 2008;101:1759-65.

16. Matsukida K, Kisanuki A, Toyonaga K, Murayama T, Nakashima H, Kumanohoso T, et al. Comparison of transthoracic Doppler echocardiography and natriuretic peptides in predicting mean pulmonary capillary wedge pressure in patients with chronic atrial fibrillation. J Am Soc Echocardiogr 2001;14:1080-7.

17. Temporelli PL, Scapellato F, Corra U, Eleuteri E, Imparato A, Giannuzzi P. Estimation of pulmonary wedge pressure by transmitral Doppler in patients with chronic heart failure and atrial fibrillation. Am J Cardiol 1999; 83:724-7.

18. Nagueh SF, Middleton KJ, Kopelen HA, Zoghbi WA, Quinones MA. Doppler tissue imaging: a noninvasive technique for evaluation of left ventricular relaxation and estimation of filling pressures. J Am Coll Cardiol 1997;30:1527-33.

19. Schiller NB, Shah PM, Crawford M, DeMaria A, Devereux R, Feigenbaum $\mathrm{H}$, et al. Recommendations for quantitation of the left ventricle by two-dimensional echocardiography: American Society of Echocardiography Committee on Standards, Subcommittee on Quantitation of TwoDimensional Echocardiograms. J Am Soc Echocardiogr 1989;2:358-67.

20. Rudski LG, Lai WW, Afilalo J, Hua L, Handschumacher MD, Chandrasekaran K, et al. Guidelines for the echocardiographic assessment of the right heart in adults: a report from the American Society of Echocardiography endorsed by the European Association of Echocardiography, a registered branch of the European Society of Cardiology, and the Canadian Society of Echocardiography. J Am Soc Echocardiogr 2010;23:685713.

21. Oyama R, Murata K, Tanaka N, Takaki A, Ueda K, Liu J, et al. Is the ratio of transmitral peak E-wave velocity to color flow propagation velocity useful for evaluating the severity of heart failure in atrial fibrillation? Circ J 2004;68:1132-8.

22. Takatsuji H, Mikami T, Urasawa K, Teranishi J, Onozuka H, Takagi C, et al. A new approach for evaluation of left ventricular diastolic function: spatial and temporal analysis of left ventricular filling flow propagation by color M-mode Doppler echocardiography. J Am Coll Cardiol 1996;27: 365-71.

23. Kidawa M, Coignard L, Drobinski G, Krzeminska-Pakula M, Thomas D, Komajda M, et al. Comparative value of tissue Doppler imaging and $\mathrm{m}$ mode color Doppler mitral flow propagation velocity for the evaluation 
of left ventricular filling pressure. Chest 2005;128:2544-50.

24. Kusunose K, Yamada H, Nishio S, Tomita N, Niki T, Yamaguchi K, et al. Clinical utility of single-beat E/e' obtained by simultaneous recording of flow and tissue Doppler velocities in atrial fibrillation with preserved systolic function. JACC Cardiovasc Imaging 2009;2:1147-56.

25. Wada Y, Murata K, Tanaka T, Nose Y, Kihara C, Uchida K, et al. Simultaneous Doppler tracing of transmitral inflow and mitral annular velocity as an estimate of elevated left ventricular filling pressure in patients with atrial fibrillation. Circ J 2012;76:675-81.

26. Rivas-Gotz C, Manolios M, Thohan V, Nagueh SF. Impact of left ventricular ejection fraction on estimation of left ventricular filling pressures using tissue Doppler and flow propagation velocity. Am J Cardiol 2003; 91:780-4.

27. Firstenberg MS, Levine BD, Garcia MJ, Greenberg NL, Cardon L, Morehead AJ, et al. Relationship of echocardiographic indices to pulmonary capillary wedge pressures in healthy volunteers. J Am Coll Cardiol 2000; 36:1664-9.
28. Tsang TS, Barnes ME, Gersh BJ, Bailey KR, Seward JB. Left atrial volume as a morphophysiologic expression of left ventricular diastolic dysfunction and relation to cardiovascular risk burden. Am J Cardiol 2002;90: 1284-9.

29. Henry WL, Morganroth J, Pearlman AS, Clark CE, Redwood DR, Itscoitz SB, et al. Relation between echocardiographically determined left atrial size and atrial fibrillation. Circulation 1976;53:273-9.

30. Sanfilippo AJ, Abascal VM, Sheehan M, Oertel LB, Harrigan P, Hughes RA, et al. Atrial enlargement as a consequence of atrial fibrillation: a prospective echocardiographic study. Circulation 1990;82:792-7.

31. Moller JE, Hillis GS, Oh JK, Pellikka PA. Prognostic importance of secondary pulmonary hypertension after acute myocardial infarction. Am J Cardiol 2005;96:199-203.

32. Neuman Y, Kotliroff A, Bental T, Siegel RJ, David D, Lishner M. Pulmonary artery pressure and diastolic dysfunction in normal left ventricular systolic function. Int J Cardiol 2008;127:174-8. 